\title{
Lost in translation: The acute care nurse practitioners' use of evidence based practice: A qualitative study
}

\author{
Steven Branham ${ }^{1}$, Rita DelloSritto ${ }^{2}$, Tara Hilliard ${ }^{1}$ \\ 1. Schools of Nursing, Texas Tech University, Lubbock Texas, USA. 2. College of Nursing, Texas Woman's University, \\ Houston Texas, USA.
}

Correspondence: Steven Branham. Address: 4429 Briarbend Drive, Houston, Texas, USA. Email:

steve.branham@gmail.com

Received: February 7, 2014

Accepted: March 28, $2014 \quad$ Online Published: April 20, 2014

DOI : $10.5430 /$ jnep.v4n6p53

URL: http://dx.doi.org/10.5430/jnep.v4n6p53

\begin{abstract}
Evidenced based practice (EBP) is a concept that has been widely enveloped by the health care industry. The benefits of EBP include cost savings, improved patient outcomes, and the translation of research findings to the bedside. In spite of the industry's acceptance of EBP, the literature is devoid of studies exploring the ways in which EBP is integrated into daily practice. This study used a qualitative approach to study the daily use of EBP by Acute Care Nurse Practitioners (ACNPs) which have far reaching implication for all health care providers who use EBP. A total of 10 practicing ACNPs from two large teaching and one community hospitals in a major, southern city in the United States, were interviewed. The transcripts were reviewed and thematic descriptors were identified. The ACNPs were overwhelmingly supportive of the use of EBP. EBP was the preferred method of delivering care, yet it was not regarded as a blanket prescription for care. A wide range of mitigating factors were identified, mandating careful consideration prior to applying EBP recommendations. The mitigating factors included: (1) beyond the barriers and balancing influencing factors, (2) to trust or not to trust, (3) balancing clinical knowledge and evidence in daily practice, (4) the patient is always right. In other words, balancing patient needs and desires often precluded the implementation of EBP tenets. This study's findings document the need for further study, improved instrument development, and educational initiatives that focus on the real world practice of integrating EBP among ACNPs and all health care providers.
\end{abstract}

\section{Key words}

Evidenced-based practice, Acute care nurse practitioners, Nurse practitioner, Utilization of evidenced-based practice

\section{I ntroduction}

The acute care nurse practitioner (ACNP) role is the newest in the subspecialty domains for nurse practitioners. The first United States (US) national certification for ACNPs took place in $1996{ }^{[1]}$. The American Nurses Credentialing Center (ANCC) reports that there are over 3,466 nationally certified ACNPs as of April 2010, with 259 residing in the state of Texas. An essential component of the ACNP role is to conduct or participate in practice related research and to evaluate and apply research findings in practice. Research efforts focused on the role of the advanced practice nurse (APN) are described as falling into three research domains which include: outcomes, innovations in nursing care, and to validate multiple complexity of nursing/APN roles ${ }^{[2]}$. Today's health care environment demands all APNs play a role in conducting, translating, integrating, and utilizing research in daily practice. 
Two national published documents, Scope and Standards for the Acute Care Nurse Practitioner (American Association of Critical Care Nurses [AACN ${ }^{[3]}$, and Acute Care Nurse Practitioner Competencies, as outlined by National Panel for Adult-Gerontology Acute Care Nurse Practitioner Competencies ${ }^{[4]}$, include research utilization and participation as components of the scope of practice for the ACNP. However, it is unclear to what degree ACNPs are involved in research activities, ranging from basic research to evidence-based practice (EBP). Kleinpell, Ely and Grabenkort ${ }^{[5]}$ noted that few studies have been conducted on ACNPs' use of EBP.

The concept of EBP has gained wide acceptance in the health care industry. Early definitions of EBP were focused primarily on research utilization. Research utilization is not the same as EBP, although the terms are often used interchangeably, many health care providers do not recognize the differences between the terms ${ }^{[6]}$. Research utilization is the narrower term, and refers to translating research findings into practice based on the level of available evidence. EBP is a broader term, and incorporates the conscious, explicit, and prudent use of current best evidence in making decisions about the care of patient and families ${ }^{[7]}$. EBP goes beyond research utilization, in that not only are research findings considered, but other factors such as clinical expertise, patient preferences/circumstances, clinical settings, and available resources are significant determinants of care ${ }^{[6]}$. The current universally accepted definition of EBP incorporates the integration of the best research evidence coupled with clinical expertise and patient values ${ }^{[8]}$. EBP has consistently been shown to improve patient outcomes ${ }^{[9]}$, but nurses often lack the time and access to knowledge sources such as databases and journals. Further, they often lack the ability to critically evaluate evidence relevant to the practice setting ${ }^{[10]}$. These factors are important at a time when the concept of EBP have been embraced by major organizations such as the Institute of Medicine ${ }^{[11]}$, the American Nurses Credentialing Center ${ }^{[11]}$, and the Joint Commission on Accreditation of Healthcare Organizations ${ }^{[12,13]}$. All have endorsed EBP as a method to improve the quality of health care.

A growing body of literature has focused on the facilitators as well as barriers to research utilization and EBP ${ }^{[14-17]}$. Few reports appear in the literature that are directly related to the nurse practitioner's use of EBP. In a study utilizing nurse practitioner educators, numerous barriers were reported to EBP use, such as resource availability (time/money), oppositional clinical mind set, research training based on research production not utilization, lack of access to data, and lack of mentorship ${ }^{[18]}$. These factors serve to inhibit maximal implementation of EBP, as well as practice based changes ${ }^{[19,20]}$. To date, it is not known if ACNP experience the same barriers as other nurses, or to what degree their practice is evidenced based. This study examines EBP use among ACNPs in their daily practice.

\section{Method}

This phenomenological, qualitative study was approved by Texas Woman's University Institutional Review Board (IRB). Nationally certified ACNPs, actively engaged in clinical practice, were recruited for this study using a combination of purposeful and snowball sampling methods. After obtaining informed consent, audio taped interviews were conducted. Data saturation was obtained after nine interviews, but one additional participant was added based on unique attributes possessed.

Transcripts were made of each semi-structured interview and reviewed for accuracy and rigor. Merleau-Ponty's approach to phenomenology reduction served as the theoretical underpinnings of this study of ACNPs' understanding and use of EBP in their daily professional lives and collective world view ${ }^{[21]}$. The identified themes provide rich insight into the individuals' work life experience as an ACNP, and the associated barriers and facilitators to the use of EBP in their day to day practice. EBP has far reaching implications for all health care professionals practicing in the evidence age.

\section{Setting}

The fourth largest city in the United States served as the geographic boundary for this study. The city is composed of 2,001,430 people, of which $49.9 \%$ are male and $51.1 \%$ are female. Nationally certified ACNPs who work or reside in the Houston area and practice in a clinical role, full time or part time, were invited to participate in the study. At the conclusion 
of the interview, the ACNPs were invited to refer colleagues to participate in the study. A total of 10 participants were enrolled in the study from three different Houston, Texas hospitals. The participants' hospital practice sites included two university-affiliated teaching hospitals of 950 and 1,250 beds, respectively. The third hospital was a suburban community network-affiliated hospital with 300 beds.

Demographic variables were analyzed by using IBM ${ }^{\circledR}$ SPSS ${ }^{\circledR}$ Statistical Package Version 19 . The sample consisted of eight female and two male ACNPs of whom nine where Caucasian and one African American. Marital status was reported as, five married, three single and two divorced. The majority of the sample participants were married Caucasian females whose ages ranged from 25 to 60 years $(M=43.1, S D=11.15)$. The sample was well educated, with five participants holding masters' degrees, two with post-masters' degrees in nursing, and three with a Doctorate of Nursing Practice. Additionally, four held baccalaureate degrees outside the field of nursing. One participant entered the field of nursing with an entry-level master's degree, graduating as both an RN and an ACNP, resulting in a range of nursing experience from 0 to 30 years $(M=15.85, S D=9.357)$. The practice areas specific to this ACNP group include five participants practicing in critical care, cardiology, or general ward. Five cared for a mixture of critical care and general ward patients.

\section{Results}

All ACNP participants perceived benefits to the use of EBP in daily practice. One issue surfaced repeatedly, EBP/Clinical Practice Guidelines (CPG) could not be applied universally to every patient situation. The general consensus of the ACNPs was that as well as knowing when to apply evidence, it was equally as important to know when not to apply evidence. EBP was viewed as an important adjunctive factor when providing clinical care within imposed limits rather than a panacea for health care delivery. Evidence was a fundamental building block for care, but could not be implemented all of the time. Prior experience, institutional factors, and patient issues all must be weighed in concert with one another when applying evidence to individual patients. An ACNP, who has practiced in cardiology for seven years, summed the general views of the group stating: "You have to think. Of course. You can't just use the guideline because it's out, why would we be there? Anybody could check off a guideline. Isn't that why we're there? To think?"

The overarching theme of the study was that ACNP viewed EBP as an important adjunctive factor that brought stability and standard approaches to practice. Even though there was a strong view which supported the use of EBP there were a wide range of mitigating factors which the clinician had to consider before applying EBP/CPGs. The mitigating factors (figure 1) which ACNPs commonly cited included: 1) beyond the barriers and balancing factors; 2) to trust or not to trust; 3) balancing clinical knowledge and evidence; and 4) patient-centered factors.

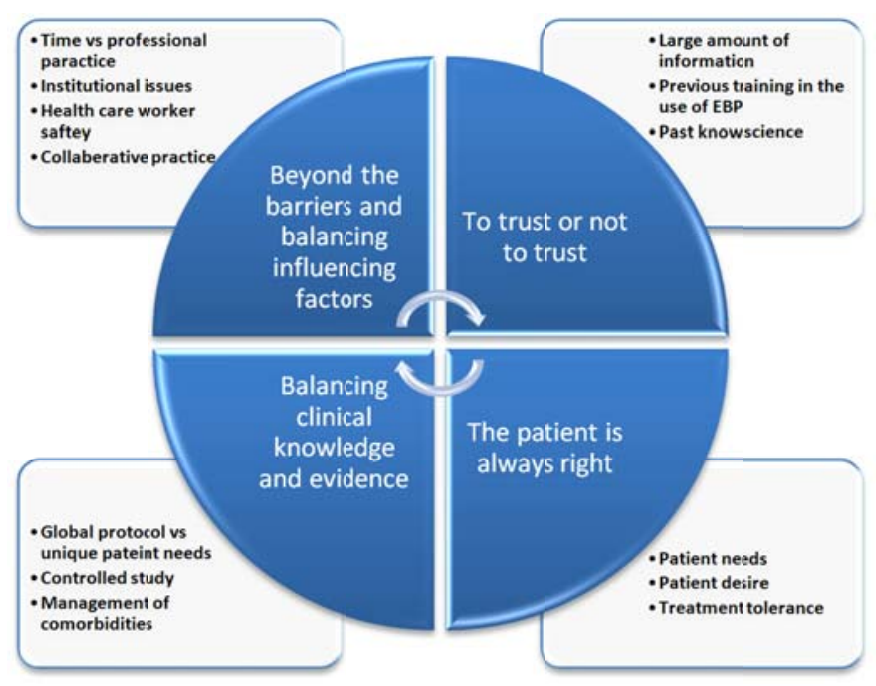

Figure 1. Acute Care Nurse Practitioner identified themes impacting the use of EBP in daily practice 


\subsection{Theme one: Beyond the barriers and balancing influencing factors}

The participants cited a number of factors that served as barriers to implementation of EBP. One, the lack of time, was a commonly cited factor in the literature coupled with the vast amounts of information produced. However, other factors were ones that affected the quality of patient care, and included worker safety and collaborative relationships. Some of these factors led the participants to make a conscious decision to deviate from an evidence based protocol. These factors were termed balancing factors and were those factors identified by the participants as influencing their decision-making process when using EBP. These factors were grouped into three categories: time/professional balance, institutional issues, and collaborative practice issues.

\subsubsection{Time/ professional practice balance}

Four ANCP participants cited the onus of time as a major barrier to the use of EBP. Time limits were further compounded by the sheer volume of information that an ACNP encounters while balancing other aspects of professional practice. In spite of time being a factor, ACNPs realized such a great benefit to the use of EBPs, methods were found to succinctly incorporate and use the data. ACNP 2 made the representative statement: "I need to do more research in different topics but trying to find the time to do it at work..... You try to read an article, but you get pulled in 12 different directions.”

\subsubsection{I nstitutional issues}

The nature of ACNP practice in the hospital setting is collaborative. Thus, it is heavily dependent on practice patterns, preferences of others, and institutional factors. ACNPs report institutional issues which impact EBP use as policies, readiness, and health care worker safety. Seven of the 10 ACNPs described institutional issues as a major barrier to the implementation of EBP. Institutional policy and availability of resources do not change as quickly as EBP changes are mandated.

ACNP 8 describes how there is often a delay in adapting new guidelines, stating: "You know, the newer guidelines talk about using ultrasound for line insertion. And one facility that I worked ... it was instituted very readily; [here] it was a little slower to catch on. So I think within just the last 2 to 3 months it became required to use ultrasound at the bedside for line insertion that has been out for several years ... But that took some time for them to incorporate it.”

\subsubsection{Health care worker safety}

The safety of health care workers has not been considered in the past as a factory which might mitigate the use of EBP. ACNP 3 related how nurses are reluctant to using draw sheets to assist with patient bed mobility. Current practice is not to use them due to the skin shearing that can result. ACNP 3's feeling is that with obese patients, it might be the safest way to move a patient while reducing the risk of back injuries to the health care workers: "Sometimes you have to use common sense.”

\subsubsection{Collaborative practice issues}

Working with other people was also cited as a factor of the use of EBP in practice. Even though ACNPs provide direct patient care, the care is typically provided as part of a collaborative team. The responsibility to implement EBP/CPG is vested with all team members. At times, there are differences of opinion among the team, and the ACNP often yields the EBP treatment plan to those who have greater expertise or control over patient care. Secondly, influencing the practice patterns of others is not always possible. ACNP 6 noted that there is often more than one correct way to treat most patient conditions. As a collaborative team member, maintaining political boundaries can impact EBP implementation by ACNPs and all health team members.

\subsection{Theme two: To trust or not to trust}

The ACNP participants reported that they use a wide range of information sources largely improving time use efficiency while acquiring EBP knowledge. The reading of formal research was reserved for areas in which they lack familiarity. The ACNPs all reported formal training in evaluating research and had firm methods for stratifying research validity. The sheer 
volume of information that is available, coupled with the ACNPs' knowledge needs in so many areas, leads them to seek information sources that are brief and factual. A strong emphasis was placed on trusted filtered information in the form of protocols/CPGs which were reserved for areas of unfamiliarity. Web browsers were used for general information (Google, Google Scholar, and Wikipedia) with a preference for medically filtered databases such as PubMed and Medline. Other online textbooks and references sited included: Harrisons Internal Medicine; Stat! Ref, Lexi-com; Washington Manual; and Braunwald' s Cardiology Textbook; as well as selected hand held device applications (Epocrates, Micromedex, Med-calcs, History and Physical, Up-to date). When viewing a wide range of information sources, the ACNPs globally issued a precautionary note: Textbooks and EBP guidelines are developed from past known science. If one bases practice solely on those sources, patients may not be availed the potential benefits of new novel drugs and treatments when compared to currently published recommendations.

\subsection{Theme three: Balancing clinical knowledge and evidence}

The application of evidence to the care of patients requires a great deal of skill on the part of the ACNP and health care providers alike. ACNPs tailor care to individual patients and, at times, elect to suspend all or parts of protocols/CPGs based on unique patient situations or preferences. EBP protocols are largely based on research findings from well controlled studies. The controls in place during the research phase are not in place when being implemented by the ACNPs. For effective implementation, clinical judgment often was utilized to safely select the aspects of the protocol that produced the greatest benefit with minimal risk. Another important finding, noted by the group, was related to the management of co-morbidities. The research findings used to develop EBP protocols often do not include the plethora of disease processes with which patients present. The ACNP clinician must balance all the unique disease processes with which the patients present while developing the treatment plan. For the ACNP, "gold standards" are not so golden when considering the myriad of factors confronting the beside clinician.

One ACNP presented a primary example of a time when "clinical courage" was employed over the strict application of an EBP protocol. The ACNP related that a strict EBP glucose management protocol was in use in the ICU. The target was to have all post-operative patients' glucose levels below $120 \mathrm{mg} / \mathrm{dl}$ with use of insulin infusions. Over time, the ACNP noticed many patients were experiencing life treating episodes of hypoglycemia. In the ACNP's estimation, the protocol was too "tight" and predisposed patients to severe hypoglycemia events. Based on the concern for patient safety, the ACNP amended the recommendation to have a target glucose level of 160 , which resulted in fewer incidences of hypoglycemia. The ACNP's diverse clinical knowledge led the ACNP to believe that the research based controls that were used in the protocol development where not safely attainable in bedside practice. It was interesting to note that those guidelines were later updated, opting for higher glucose set points. In this case, the clinician was right, and took steps before consensus groups could make the necessary changes based on the evolving reports of adverse patient events.

\subsection{Theme four: The patient is always right}

In an effort to balance the patients' needs and the desired patient needs, the ACNPs in this study reported a wide range of patient-specific factors that must be considered when making treatment decisions. The most commonly occurring issues included treatment intolerances, costs, and patient acceptance of the burden of treatment. It is easy to see that treatment intolerance and the cost of treatment could be prohibitive issues when implementing EBP practices. Patients often have sociocultural biases that must be factored in when deciding to accept or reject the burden of treatment. ACNP 5 provided an outstanding example of a patient opting out of EBP recommendations out of feared safety issues. The patient was a 45 year old man who went into atrial fibrillation that could not be converted to a normal rhythm. The EBP guidelines stated that he should be placed on Coumadin to prevent adverse events. When the patient reviewed the side effects he said. "I install satellite dishes and cannot quit my job. I can’t safely do this.” He was placed on aspirin alone, as a safer option, given his occupational risks. Ultimately, patients decide when to accept treatment and whether the treatment options are congruent with their individual needs. 


\section{Discussion}

The use of phenomenological investigative methods provides the first known insight into the essence of EBP use by ACNPs, outlining a collective worldview of EBP use in clinical practice. These findings are specific to ACNPs, but they have far-reaching ramifications for all health care professionals who incorporate EBP into their daily practices. The concept of EBP has gained widespread acceptance that most often incorporates one tenet of EBP, which is the use of the best available evidence. Equally important components of the definition are the use of patient preferences and clinical judgment, which are woefully under-reported in the literature.

ACNPs view EBP as an integral part of patient care. To maximally integrate EBP, prompt access to information from a variety of sources is essential. When applying EBP clinically, ACNPs must consider any number of factors and attenuate the application to specific patient requirements. Institutional factors, such as availability of service and collaborative team member input, are essential components of the implementation process. The often less credited aspects of clinical decision making and patient preferences are key components when applying EBP to individual patient needs. Clinicians must, at times, go against conventional wisdom and courageously challenging the strict application of research when clinical derived knowledge denotes more cautious application should be used. Further consideration must be given to patient derived factors, such as treatment cost, ill effects of treatment, and individual patient preference. Patient preference is an EBP application filter that remains supreme.

Globally for ACNPs, as well as other health care providers, reimbursement standards, which mandate $100 \%$ compliance for optimal payment levels, are short sighted and do not take into account all the factors required in informed clinical decision making and treatment plan development. Educational initiatives should focus members of the interdisciplinary team to consider all aspects of the EBP paradigm, and not solely on research utilization.

\section{Conclusions}

Based on the results of this study, the researchers concluded that ACNPs have respect for EBP. The practitioners incorporate the use of EBPs in their daily practice. Although, EBPs were felt to be an important adjunct to daily practice, it is paramount to balance clinical knowledge, professional responsibilities, institutional constrains, as well as patient needs when developing plans of care. Individual providers' must have the latitude to deliver safe and effective care tailored to the patient's needs, desirers, and lifestyle. Practice protocols and guideline provide the most efficient way for ACNPs to monitor and promptly integrate evidence into practice.

Conversely, it was found that there was no difference in evidence based practice use in ACNPs who had a dedicated evidenced based practice course when compared to those who had it integrated throughout the curriculum. Regardless of instructional methodology, it is important for all health care providers to have formal training in the use of EBP. Ultimately, the goal of ACNP practice is to attain the best clinical outcome for the individual patient and family.

\section{References}

[1] Kleinpell RM. Acute care nurse practitioner practice: Results of a 5-year longitudinal study. Am J Crit Care. 2005; 14(3): 211-221. Available from: http://ezproxy.twu.edu:2048/login?url=http://search.ebscohost.com/login.aspx?direct=true\&db=rzh\&AN=2005106294\&site=eh ost-live\&scope=site. PMid:15840895

[2] Facchiano L, Snyder C, Hoffman. Evidence-based practice for the busy nurse practitioner: Part two: Searching for the best evidence to clinical inquiries. J Am Acad Nurse Pract. 2012; 24(11): 640-648. Available from: http://ezproxy.twu.edu:2048/login?url=http://search.ebscohost.com/login.aspx?direct=true\&db=rzh\&AN=2011716398\&site=eh ost-live\&scope=site. PMid:23088695 http://dx.doi.org/10.1111/j.1745-7599.2012.00749.x 
[3] Bell L, ed. AACN scope and standards for acute care nurse practitioner practice. Aliso Viejo, Ca: American Association of Critical-Care Nurses; 2012. ISBN 978-0-945812-01-2.

[4] Adult-gerontology acute care nurse practitioner competencies. Washington, DC.: American Association of Colleges of Nursing; 2012.

[5] Kleinpell RM. Acute care nurse practitioner practice: Results of a 5-year longitudinal study. Am J Crit Care. 2005; 14(3): 211-221. Available from: http://ezproxy.twu.edu:2048/login?url=http://search.ebscohost.com/login.aspx?direct=true\&db=rzh\&AN=2005106294\&site=eh ost-live\&scope=site. PMid:15840895

[6] Polit DF, Beck CT, eds. Nursing research generating and assessing evidence for nursing practice. Ninth ed. Philadelphia PA: Lippincott Williams \& Wilkinis; 2012.

[7] Sackett DL, Rosenberg WM, Gray JA, Haynes RB, Richardson WS. Evidence based medicine: What it is and what it isn't. 1996. Clin Orthop Relat Res. 2007; 455: 3-5. PMid:17340682

[8] David L. Sackett, DL, Straus SE, Richardson WS, Rosenberg W, Haynes B, ed. Evidence-based medicine: how to practice and teach EBM. 2nd ed. United Kingdom: Churchill, Livinston; 2000.

[9] Malloch K, Porter-O'Grady T. Introduction to evidence-based practice in nursing and health care. Sidburry, Massachusetts: Jones and Barltett's; 2010

[10] Hart P, Eaton L, Buckner M, et al. Effectiveness of a computer-based educational program on nurses' knowledge, attitude, and skill level related to evidence-based practice. Worldviews Evid Based Nurs. 2008; 5(2): 75-84. http://dx.doi.org/10.1111/j.1741-6787.2008.00123.x

[11] IOM report on the future of nursing: Leading change, advancing health. MEDSURG MATTERS. 2011; 20(1): 3-3. Available from:

http://ezproxy.twu.edu:2048/login?url=http://search.ebscohost.com/login.aspx?direct=true\&db=rzh\&AN=2010978901\&site=eh ost-live\&scope=site.

[12] Rosswurm MA, Larrabee JH. Clinical scholarship. A model for change to evidence-based practice. IMAGE J NURS SCHOLARSH. 1999; 31(4): 317-322. Available from: http://search.ebscohost.com/login.aspx?direct=true\&db=rzh\&AN=2000009461\&site=ehost-live

[13] Long KA. The institute of medicine report health professions education: A bridge to quality. POLICY POLIT NURS PRACT. 2003; 4(4): 259-262. http://dx.doi.org/10.1177/1527154403258304

[14] Funk SG, Tornquist EM, Champagne MT. Barriers and facilitators of research utilization: An integrative review. Nurs Clin North Am. 1995; 30(3): 395-407. PMid:7567566

[15] LaPierre E, Ritchey K, Newhouse R. Barriers to research use in the PACU. Journal of perianesthesia nursing: official journal of the American Society of PeriAnesthesia Nurses. 2004; 19(2): 78-83. http://dx.doi.org/10.1016/j.jopan.2004.01.001

[16] Parahoo K, Mccaughan EM. Research utilization among medical and surgical nurses: A comparison of their self reports and perceptions of barriers and facilitators. J Nurs Manag. 2001; 9(1): 21-30. http://dx.doi.org/10.1046/j.1365-2834.2001.00237.x

[17] Retsas A. Barriers to using research evidence in nursing practice. J Adv Nurs. 2000; 31(3): 599-606. http://dx.doi.org/10.1046/j.1365-2648.2000.01315.x

[18] Melnyk BM, Fineout-Overholt E, Feinstein NF, Sadler LS, Green-Hernandez C. Nurse practitioner educators' perceived knowledge, beliefs, and teaching strategies regarding evidence-based practice: Implications for accelerating the integration of evidence-based practice into graduate programs. J Prof Nurs. 2008; 24(1):7-13. http://dx.doi.org/10.1016/j.profnurs.2007.06.023

[19] Butler K, Diane. Nurse practitioners and evidence-based nursing practice. CLIN SCHOLARS REV. 2011; 4(1): 53-57. Available from: http://ezproxy.twu.edu:2048/login?url=http://search.ebscohost.com/login.aspx?direct=true\&db=rzh\&AN=2011202992\&site=eh ost-live\&scope=site. http://dx.doi.org/10.1891/1939-2095.4.1.53

[20] Kleinpell RM, Ely EW, Grabenkort R. Nurse practitioners and physician assistants in the intensive care unit: An evidence-based review. Crit Care Med. 2008; 36(10): 2888-2897. http://dx.doi.org/10.1097/CCM.0b013e318186ba8c

[21] 21 Merleau-Ponty M. Phenomenology of perception. Rev ed. London; Atlantic Highlands, N.J.: Routledge \& K. Paul; Humanities Press; 1981; 1962: 466. 